\title{
Magnetization dynamics in clean and disordered spin-1 XXZ chains
}

\author{
Jonas Richter, ${ }^{1, *}$ Niklas Casper, ${ }^{2}$ Wolfram Brenig, ${ }^{2}$ and Robin Steinigeweg ${ }^{1, \dagger}$ \\ ${ }^{1}$ Department of Physics, University of Osnabrück, D-49069 Osnabrück, Germany \\ ${ }^{2}$ Institute for Theoretical Physics, Technical University Braunschweig, D-38106 Braunschweig, Germany
}

(Dated: October 16, 2019)

\begin{abstract}
We study spin transport in the one-dimensional anisotropic $S=1$ Heisenberg model. Particular emphasis is given to dynamics at infinite temperature, where current autocorrelations and spatiotemporal correlation functions are obtained by means of an efficient pure-state approach based on the concept of typicality. Our comprehensive numerical analysis unveils that high-temperature spin transport is diffusive in the easy-axis regime for strong exchange anisotropies. This finding is based on the combination of numerous signatures, such as (i) Gaussian spreading of correlations, (ii) a time-independent diffusion coefficient, (iii) power-law decay of equal-site correlations, (iv) exponentially decaying long-wavelength modes, and (v) Lorentzian line shapes of the dynamical structure factor. Moreover, we provide evidence that some of these signatures are not exclusively restricted to the infinite-temperature limit, but can persist at lower temperatures as well, where we complement our results by additional quantum Monte Carlo simulations of large systems. In contrast to the easy-axis regime, we show that in the case of an isotropic chain, the signatures (i) - (v) are much less pronounced or even entirely absent, suggesting the existence of anomalous spin transport despite the nonintegrability of the model. Eventually, upon introducing a random on-site magnetic field, we observe a breakdown of diffusion and distinctly slower dynamics. In particular, our results exhibit qualitative similarities to disordered spin-1/2 chains and might be consistent with the onset of many-body localization in the $S=1$ model for sufficiently strong disorder.
\end{abstract}

\section{INTRODUCTION}

Fundamental aspects about the emergence of thermodynamic behavior in closed quantum many-body systems have recently attracted rejuvenated interest $[1-3]$. While there has been immense progress due to, e.g., theoretical concepts such as the eigenstate thermalization hypothesis [4-6], large-scale numerical simulations [7], as well as the advance of new experimental platforms [8, 9], there are still challenging problems calling for a deeper understanding. For instance, a particularly intriguing question is whether or not conventional hydrodynamic transport, i.e., diffusion, can arise in isolated quantum systems undergoing solely unitary time evolution [10].

Intimately related to this question is the distinction between integrable and nonintegrable models. On the one hand, integrable models are characterized by a macroscopic number of (quasi)local conservation laws which can lead to anomalous thermalization $[11,12]$ and ballistic transport [13-15]. As a consequence, diffusion is generally not expected to occur in these systems. Nevertheless, while subleading diffusive corrections have been established within the framework of generalized hydrodynamics [16-18], there is also clear numerical evidence for diffusive transport in certain integrable models and parameter regimes [19-27].

On the other hand, integrability is rather the exception than the rule and can be broken in numerous ways, e.g., spin-phonon coupling [28, 29], long-range interactions [30, 31], impurities [32, 33], or disorder [34]. For

\footnotetext{
* jonasrichter@uos.de

† rsteinig@uos.de
}

such nonintegrable models, Drude weights are expected to vanish [15] and diffusion might emerge, e.g., due to quantum chaos [35]. Although much progress in understanding the emergence of diffusive hydrodynamics has been recently made in the study of random unitary circuit models [36-38], observations of genuine diffusion in realistic systems are comparatively rare [22, 39-43]. In particular, it is still an open question if diffusion is indeed a generic feature for all integrability-breaking perturbations (note that counterexamples have been proposed [33]). Moreover, answering this question is also very challenging due to the tremendous numerical requirements which arise in the study of transport in quantum many-body systems, such as the exponential growth of the Hilbert-space dimension as well as the necessity to study long time scales.

In this context, we consider yet another nontrivial way to break integrability, i.e, the consideration of a larger spin quantum number $S>1 / 2[44,45]$. In particular, we study spin transport in the one-dimensional $S=1$ XXZ model, using an efficient numerical approach based on the concept of quantum typicality [46-56]. Summarizing our main results, we unveil that high-temperature spin transport is diffusive in the easy-axis regime of large anisotropies. This finding is based on the combination of numerous signatures, such as (i) Gaussian spreading of correlations, (ii) a time-independent diffusion coefficient, (iii) power-law decay of equal-site correlations, (iv) exponentially decaying long-wavelength modes, and (v) Lorentzian line shapes of the dynamical structure factor. Moreover, we provide evidence that some of these signatures are not exclusively restricted to the infinitetemperature limit, but can persist at lower temperatures as well, where we complement our results by additional 
quantum Monte Carlo simulations of large systems. In contrast to the easy-axis regime, we show that in the case of an isotropic chain, the signatures (i) - (v) are much less pronounced or even entirely absent, suggesting the existence of anomalous spin transport despite the nonintegrability of the model. Eventually, upon introducing a random on-site magnetic field, we observe a breakdown of diffusion and distinctly slower dynamics. In particular, our results exhibit qualitative similarities to disordered spin-1/2 chains and might be consistent with the onset of many-body localization in the $S=1$ model for sufficiently strong disorder.

This paper is structured as follows. We introduce the model in Sec. II and define the observables in Sec. III. In Sec. IV we explain our numerical approach, and we present our results in Sec.V. We conclude and summarize in Sec. VI.

\section{MODEL}

We study the one-dimensional $S=1 \mathrm{XXZ}$ model with periodic boundary conditions, described by the Hamiltonian

$$
\mathcal{H}=J \sum_{l=1}^{L}\left(S_{l}^{x} S_{l+1}^{x}+S_{l}^{y} S_{l+1}^{y}+\Delta S_{l}^{z} S_{l+1}^{z}\right),
$$

where the $S_{l}^{x, y, z}$ are spin-1 operators at lattice site $l$, $J=1$ denotes the antiferromagnetic exchange constant (and sets the energy scale throughout this paper), $L$ is the number of sites, and $\Delta>0$ is the anisotropy in $z$ direction. In contrast to its spin- $1 / 2$ counterpart, the Hamiltonian (1) is nonintegrable for $S=1$ [45, 57].

The spin-1 chain (1) is a fundamental model of lowdimensional quantum magnetism and is realized to good quality in numerous materials. As a consequence, its thermodynamic and its dynamical properties have been intensively scrutinized by theoretical [45, 58-63] and experimental techniques [64-69]. Moreover, various modifications to the bare Hamiltonian (1) have been explored as well, such as, e.g., single-ion anisotropy and external magnetic fields [70-74]. While experiments have reported on the existence of diffusive spin and energy transport in spin-1 compounds [68, 69], theoretical studies have given contradictory results and argued for diffusive $[60,75,76]$ but also ballistic transport [77, 78]. In this context, it is important to note that Refs. [75, 77, 78] have in fact considered the non-linear sigma model as the effective low-energy description of Eq. (1), where additional conservation laws might have an impact on the transport properties.

While the focus of this paper is on $\operatorname{spin} S=1$, it is instructive to briefly recap the nature of spin dynamics in the integrable $S=1 / 2$ version of Eq. (1). On the one hand, for $\Delta<1$, the spin- $1 / 2$ chain features a finite Drude weight, i.e., ballistic transport [14, 82, 83]. On the other hand, for $\Delta>1$, the Drude weight vanishes and clean signatures of diffusion have been observed
[19-24]. While the situation is arguably most controversial for $\Delta=1$, recent works advocate the presence of superdiffusion at the isotropic point at high temperatures [24, 79, 80]. In this context, it is an intriguing question if normal diffusion generically occurs in the onedimensional XXZ model (i.e. for all $\Delta$ ) upon considering the larger spin quantum number $S=1$, both at infinite and also finite temperatures (see also Ref. [81]). We explore this question in Secs. V A and VB.

In Sec. VC, we additionally study the spin-1 XXZ chain in the presence of a random magnetic field, i.e., the Hamiltonian (1) is modified according to

$$
\mathcal{H}=J \sum_{l=1}^{L}\left(S_{l}^{x} S_{l+1}^{x}+S_{l}^{y} S_{l+1}^{y}+\Delta S_{l}^{z} S_{l+1}^{z}+h_{l} S_{l}^{z}\right)
$$

where the on-site magnetic fields $h_{l} \in[-W, W]$ are drawn at random from a uniform distribution, with $W \geq 0$ setting the magnitude of disorder.

Once again, let us briefly reiterate the case of $S=1 / 2$. In fact, the disordered spin-1/2 Heisenberg chain is a central model to study the disorder-driven transition between a thermal phase $\left(W<W_{c}\right)$ and a many-bodylocalized (MBL) phase $\left(W>W_{c}\right)$, where $W_{c}$ is a critical disorder strength $[84,85]$. This MBL phase is characterized by, e.g., a vanishing dc conductivity [86, 87], area-law entanglement of eigenstates [88, 89], the emergence of a set of local integrals of motion [90, 91], as well as the logarithmic growth of entanglement with time [92, 93]. While ground-state properties of disordered spin-1 systems have been studied before [94, 95], their dynamics has remained largely unexplored. Therefore, the present paper attempts to elucidate the effect of disorder on spin dynamics in the anisotropic spin-1 chain.

\section{OBSERVABLES}

Let us now introduce the quantities which are studied in this paper. In particular, we discuss how diffusive transport can be detected based on these quantities.

\section{A. Current autocorrelations and transport coefficients}

Since total magnetization is conserved for all choices of $\Delta$ and $W$, the spin current $j=\sum_{l} j_{l}$ is well defined via a lattice continuity equation, $\partial_{t} S_{l}^{z}=i\left[\mathcal{H}, S_{l}^{z}\right]=j_{l-1}-j_{l}$, and takes on the form [96]

$$
j=J \sum_{l=1}^{L}\left(S_{l}^{x} S_{l+1}^{y}-S_{l}^{y} S_{l+1}^{x}\right)
$$

Within linear response theory, transport properties are related to current-current correlation functions evaluated 
in equilibrium,

$$
\langle j(t) j\rangle=\frac{\operatorname{Tr}\left[e^{-\beta \mathcal{H}} j(t) j\right]}{\mathcal{Z}},
$$

where the time argument has to be understood in the Heisenberg picture $j(t)=e^{i \mathcal{H} t} j e^{-i \mathcal{H} t}, \beta=1 / T$ denotes the inverse temperature, and $\mathcal{Z}=\operatorname{Tr}\left[e^{-\beta \mathcal{H}}\right]$ is the partition function. For instance, integration of $\langle j(t) j\rangle$ yields the time-dependent diffusion coefficient $D(t)[97]$,

$$
D(t)=\frac{1}{\chi} \int_{0}^{t} \frac{\operatorname{Re}\left\langle j\left(t^{\prime}\right) j\right\rangle}{L} \mathrm{~d} t^{\prime}
$$

where $\chi=\lim _{q \rightarrow 0}\left\langle S_{q}^{z} S_{-q}^{z}\right\rangle$ denotes the isothermal spin susceptibility. In the case of diffusion, one expects that the current autocorrelation eventually decays to zero, such that $D(t)$ saturates at a constant plateau $D(t>$ $\tau) \approx D$ for times $t$ above the mean-free time $\tau$. Generally, however, it is important to note that $D(t)$ does not distinguish between transport channels with different behavior [19].

Furthermore, the frequency-dependent spin conductivity $\sigma(\omega)$ follows from a Fourier transform of the currentcurrent correlation function,

$$
\operatorname{Re} \sigma(\omega)=\frac{1-e^{-\beta \omega}}{\omega L} \operatorname{Re} \int_{0}^{\infty} e^{i \omega t}\langle j(t) j\rangle \mathrm{d} t
$$

and is usually decomposed into a $\delta$ function at $\omega=0$ and a regular part at $\omega \neq 0$,

$$
\operatorname{Re} \sigma(\omega)=\mathcal{D} \delta(\omega)+\sigma_{\text {reg }}(\omega),
$$

where $\mathcal{D}$ is the so-called Drude weight. If transport is diffusive, we have $\mathcal{D}=0$ and there is a well-behaved dc conductivity $\sigma_{\mathrm{dc}}=\lim _{\omega \rightarrow 0} \sigma_{\mathrm{reg}}(\omega)$. Moreover, using an Einstein relation, this dc conductivity is connected to the diffusion constant according to [97],

$$
D=\frac{\sigma_{\mathrm{dc}}}{\chi}
$$

\section{B. Spin density correlations}

In addition to current dynamics, we also study the dynamics of spatio-temporal correlation functions $C_{l, l^{\prime}}(t)$ defined as,

$$
C_{l, l^{\prime}}(t)=\left\langle S_{l}^{z}(t) S_{l^{\prime}}^{z}\right\rangle=\frac{\operatorname{Tr}\left[e^{-\beta \mathcal{H}} S_{l}^{z}(t) S_{l^{\prime}}^{z}\right]}{\mathcal{Z}} .
$$

For the particular case of $\beta \rightarrow 0$, these correlations realize a $\delta$-peak profile at time $t=0$, or in other words, spins at different lattice sites are uncorrelated at infinite temperature,

$$
C_{l, l^{\prime}}(t=0)=\left\{\begin{array}{ll}
\chi>0, & l=l^{\prime} \\
0, & l \neq l^{\prime}
\end{array},\right.
$$

with $\chi=2 / 3$ for $\beta=0$ and $S=1$. For times $t>0$, however, correlations start to build up and the initial $\delta$ peak will spread over the system. Specifically, in the case of diffusion, this spreading yields a Gaussian density profile [23, 42],

$$
C_{l, l^{\prime}}(t) \propto \exp \left[-\frac{\left(l-l^{\prime}\right)^{2}}{2 \Sigma(t)^{2}}\right]
$$

where the spatial variance $\Sigma(t)^{2}$ is generally given by

$$
\Sigma(t)^{2}=\sum_{l=1}^{L} l^{2} \delta C_{l, l^{\prime}}(t)-\left(\sum_{l=1}^{L} l \delta C_{l, l^{\prime}}(t)\right)^{2}
$$

with $\delta C_{l, l^{\prime}}(t)=C_{l, l^{\prime}}(t) / \chi$ and $\sum_{l} \delta C_{l, l^{\prime}}(t)=1$. Due to continuity, this spatial variance is also related to the already mentioned diffusion coefficient [98-100],

$$
\frac{\mathrm{d}}{\mathrm{d} t} \Sigma(t)^{2}=2 D(t) .
$$

Note that Eq. (13) does not require specific assumptions on the microscopic Hamiltonian $\mathcal{H}$, apart from $\left[\mathcal{H}, \sum_{l} S_{l}^{z}\right]=0$ and the system being translational invariant. Given a diffusive process, i.e., $D(t)=D=$ const., it then follows that $\Sigma(t)^{2} \propto t$. Moreover, this particular scaling of $\Sigma(t)^{2}$ also implies that the equal-site correlation $C_{l, l^{\prime}=l}(t)$ decays as a power-law

$$
C_{l, l^{\prime}=l}(t) \propto t^{-1 / 2} .
$$

Starting from the real-space correlations in Eq. (9), the respective correlation functions in momentum space follow from a lattice Fourier transform according to [101]

$$
\begin{aligned}
C_{q}(t)=\left\langle S_{q}^{z}(t) S_{-q}^{z}\right\rangle & =\frac{1}{L} \sum_{l, l^{\prime}=1}^{L} e^{i q l} e^{-i q l^{\prime}}\left\langle S_{l}^{z}(t) S_{l^{\prime}}^{z}\right\rangle \\
& =\sum_{l=1}^{L} e^{i q l}\left\langle S_{l^{\prime}+l}^{z}(t) S_{l^{\prime}}^{z}\right\rangle
\end{aligned}
$$

where the discrete momenta $q$ are defined as usual, $q=2 \pi k / L, k=0,1, \ldots, L-1$. Note that Eq. (16) is strictly valid only for translational invariant systems $(W=0)$, but might also hold approximately for $W>0$ if the $C_{l, l^{\prime}}(t)$ are averaged over sufficiently many disorder realizations, cf. Sec. IV C. In momentum space, diffusion can be characterized by the existence of a hydrodynamic regime where long-wavelength modes exhibit an exponential decay,

$$
C_{q}(t) \propto e^{-\tilde{q}^{2} D t}
$$

with $\tilde{q}^{2}=2[1-\cos (q)] \approx q^{2}$ for small $q$.

Moreover, another Fourier transform from the time to the frequency domain yields the so-called dynamical structure factor $C_{q}(\omega)$,

$$
C_{q}(\omega)=\int_{-\infty}^{\infty} e^{i \omega t} C_{q}(t) \mathrm{d} t
$$


As a direct consequence of the exponentials in Eq. (17), diffusive transport reflects itself in a Lorentzian line shape of $C_{q}(\omega)$,

$$
C_{q}(\omega) \propto \frac{1}{\omega^{2}+\tilde{q}^{4} D^{2}},
$$

for sufficiently long wavelengths.

\section{NUMERICAL APPROACH}

\section{A. Dynamical quantum typicality}

Loosely speaking, the concept of dynamical quantum typicality (DQT) states that a single pure quantum state can have the same properties as the statistical ensemble [46-56]. In practice, this fact can be exploited in order to replace the trace in Eq. (4) by a simple scalar product with two auxiliary pure states $\left|\varphi_{\beta}(t)\right\rangle,\left|\psi_{\beta}(t)\right\rangle$ such that the current autocorrelation takes on the form $[54,56,102]$

$$
\operatorname{Re}\langle j(t) j\rangle=\frac{\operatorname{Re}\left\langle\varphi_{\beta}(t)|j| \psi_{\beta}(t)\right\rangle}{\left\langle\varphi_{\beta}(0) \mid \varphi_{\beta}(0)\right\rangle}+\epsilon,
$$

with $\left|\varphi_{\beta}(0)\right\rangle=e^{-\beta \mathcal{H} / 2}|\varphi\rangle,\left|\psi_{\beta}(0)\right\rangle=j e^{-\beta \mathcal{H} / 2}|\varphi\rangle$, and

$$
|\varphi\rangle=\sum_{k=1}^{d} c_{k}\left|\phi_{k}\right\rangle
$$

Here, the reference pure state $|\varphi\rangle$ is prepared according to the unitary invariant Haar measure [103], i.e., the complex coefficients $c_{k}$ are randomly drawn from a Gaussian distribution with zero mean. The states $\left|\phi_{k}\right\rangle$ denote a complete set of basis vectors of the Hilbert space, e.g., the Ising basis. If not stated otherwise, we always consider the full Hilbert space with dimension $d=3^{L}$.

Importantly, the statistical error $\epsilon=\epsilon(|\varphi\rangle)$ in Eq. (20) scales as $\epsilon \propto 1 / \sqrt{d_{\text {eff }}}$, where $d_{\text {eff }}=\mathcal{Z} / e^{-\beta E_{0}}$ is an effective dimension and $E_{0}$ is the ground-state energy of $\mathcal{H}$ $[51,54,56,102,103]$. Thus, $\epsilon$ decreases exponentially with increasing system size and, particularly for $\beta \rightarrow 0$, the typicality approximation becomes very accurate already for moderate values of $L[56,80]$.

Completely analogous to current autocorrelations, the spatio-temporal correlations $C_{l, l^{\prime}}(t)$ for $\beta \geq 0$ can be obtained by means of a pure-state approach as well, simply by replacing $j$ with $S_{l}^{z}$ (or $S_{l^{\prime}}^{z}$ ) in and below Eq. (20). However, in the limit $\beta \rightarrow 0$, it is even possible to calculate $C_{l, l^{\prime}}(t)$ from just one auxiliary state [43, 104] (see also Appendix A),

$$
\operatorname{Re} C_{l, l^{\prime}}(t)=\operatorname{Re}\left\langle\tilde{\psi}(t)\left|S_{l}^{z}\right| \tilde{\psi}(t)\right\rangle+\epsilon,
$$

where $|\tilde{\psi}(t=0)\rangle$ is constructed according to

$$
|\tilde{\psi}(0)\rangle=\frac{\sqrt{S_{l^{\prime}}^{z}+1}|\varphi\rangle}{\sqrt{\langle\varphi \mid \varphi\rangle}},
$$

with $|\varphi\rangle$ again being randomly drawn, cf. Eq. (21). It is important to note that the operator $S_{l^{\prime}}^{z}+1$ (i) only has nonnegative eigenvalues and (ii) is diagonal in the Ising basis. Therefore, the application of the square root operation is well-defined and rather straightforward [43].

\section{B. Pure-state propagation}

In Eqs. (20) and (22), the time argument is interpreted as a property of the pure states and not of the operators anymore,

$$
|\psi(t)\rangle=e^{-i \mathcal{H} t}|\psi(0)\rangle
$$

Compared to standard exact diagonalization (ED), the main advantage of the pure-state approach stems from the fact that this time evolution can be conveniently evaluated by means of an iterative forward propagation, $|\psi(t+\delta t)\rangle=e^{-i \mathcal{H} \delta t}|\psi(t)\rangle$. Similarly, the action of $e^{-\beta \mathcal{H} / 2}$ can be generated by a forward propagation as well, but now in imaginary time. While there exist various sophisticated methods such as Trotter decompositions [105], Chebyshev expansions [106, 107], as well as Krylov-subspace techniques [108], we here apply a fourthorder Runge-Kutta algorithm where the discrete time step $\delta t$ is always chosen short enough to guarantee negligible numerical errors $[54,56]$. Since the involved operators usually exhibit a sparse matrix representation, the matrix-vector multiplications within this Runge-Kutta scheme can be implemented memory efficient, and we can treat Hilbert-space dimensions significantly larger compared to ED. (As an example, $d=3^{L} \approx 4 \cdot 10^{8}$ for $L=18$. Note that this Hilbert-space dimension would correspond to $L \approx 29$ in the case of $S=1 / 2$.)

Eventually, concerning the Fourier transforms in Eqs. (6) and (18), let us note that the integrals can in practice be evaluated only up to a finite cutoff time $t_{\max }<\infty$, giving rise to a finite frequency resolution $\delta \omega=\pi / t_{\max }$.

\section{Averaging}

In this paper, we have to differentiate between two possible types of averaging. On the one hand, our numerical approach is based on the construction of the pure state $|\varphi\rangle$, cf. (21), comprising the random coefficients $c_{k}$. Although the statistical error $\epsilon(|\varphi\rangle)$ of the typicality approximation is rather small for large $L$, the remaining error can be reduced even further by averaging over $N_{S}$ different instances of the $c_{k}$. While such a procedure is usually unnecessary for $\beta \rightarrow 0\left(N_{S}=1\right)$, it can be beneficial for temperature regimes $T \lesssim J[52,109]$.

On the other hand, in the case of a disordered model with $W>0$, all results naturally depend on the specific configuration of the random magnetic fields $h_{l}$. In order to obtain reliable results, we therefore routinely perform 

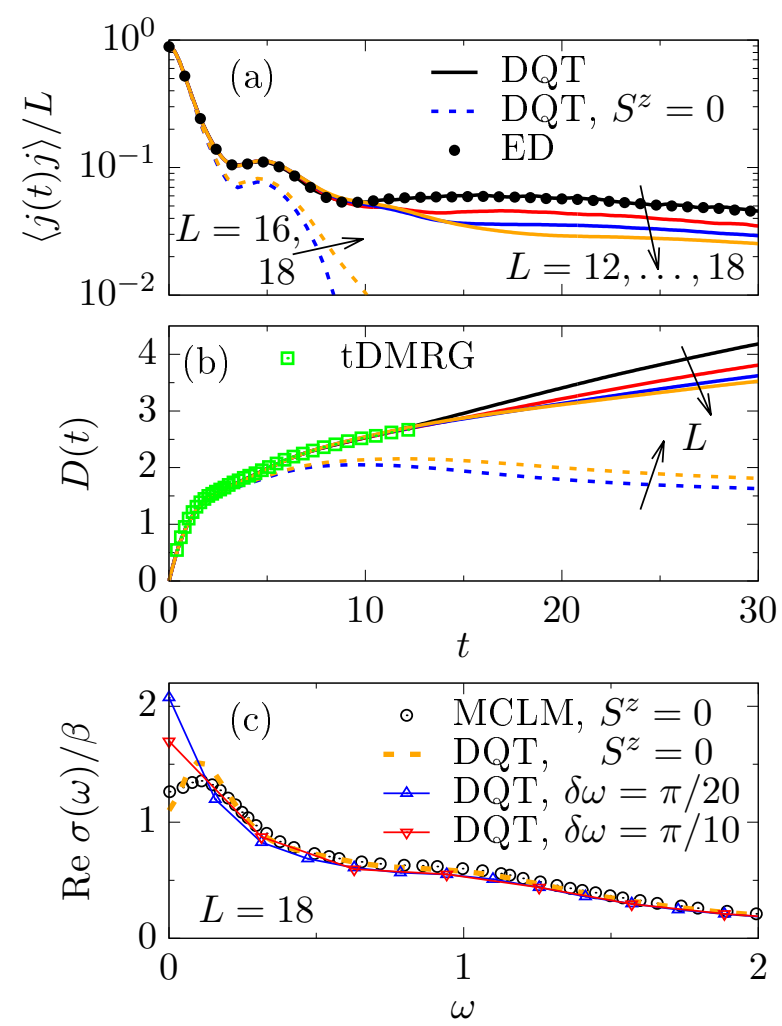

FIG. 1. (Color online) (a) Current autocorrelation $\langle j(t) j\rangle / L$ obtained for different system sizes $L=12,14,16,18$ by ED and DQT. As a comparison, we also show data for $L=16,18$ calculated in the $S^{z}=0$ subsector only. (b) Corresponding diffusion coefficient $D(t)$, cf. Eq. (5). Rescaled data from time-dependent density matrix renormalization group (tDMRG) calculations for $T=10$ are depicted [81, 116]. (c) Conductivity $\sigma(\omega)$ calculated according to Eq. (6) for frequency resolutions $\delta \omega=\pi / 10, \pi / 20$, and $\pi / 100\left(S^{z}=0\right)$. As a comparison, we depict data obtained by the microcanonical Lanczos method (MCLM) from Ref. [60]. The other parameters are $\Delta=1$ and $\beta=0$.

an averaging over a sufficiently large number $N$,

$$
C_{l, l^{\prime}}(t)=\frac{1}{N} \sum_{n=1}^{N} C_{l, l^{\prime}}^{(n)}(t)
$$

where each $C_{l, l^{\prime}}^{(n)}(t)$ is evaluated for a different random configuration of the $h_{l}$.

A useful measure, both for sampling over initial states as well as over disorder configurations, is the variance of sample-to-sample fluctuations,

$$
\Delta C_{l, l^{\prime}}(t)=\sum_{n=1}^{N_{(S)}} \frac{\left[C_{l, l^{\prime}}^{(n)}(t)\right]^{2}}{N_{(S)}}-\left(\sum_{n=1}^{N_{(S)}} \frac{C_{l, l^{\prime}}^{(n)}(t)}{N_{(S)}}\right)^{2},
$$

which will typically increase for lower temperatures $T$ and stronger disorder $W$. The value of $N_{(S)}$ has to be chosen in such a way that the error of the mean $\sqrt{\Delta C_{l, l^{\prime}}(t) / N_{(S)}}$ remains small in all cases. The above
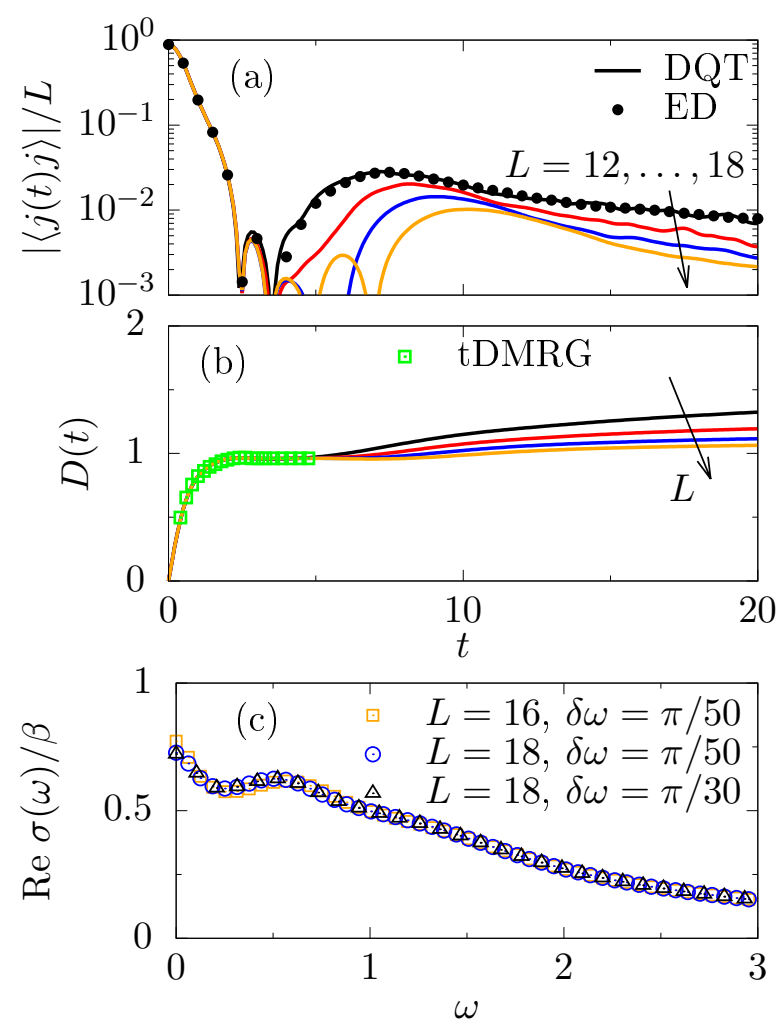

FIG. 2. (Color online) Analogous data as in Fig. 1, but now for a larger anisotropy $\Delta=1.5$. Note that we plot the absolute value $|\langle j(t) j\rangle| / L$ in (a) for better visibility. All calculations are performed in the full Hilbert space.

reasoning of course not only applies to the spatiotemporal correlations $C_{l, l^{\prime}}(t)$, but also to the current autocorrelation $\langle j(t) j\rangle$.

\section{Quantum Monte Carlo}

The quantum Monte Carlo (QMC) method is based on the stochastic series expansion (SSE) [110-112], which uses importance sampling of the high-temperature series expansion of the partition function with a truncation of the sum to order $M$,

$$
\mathcal{Z}=\sum_{\alpha} \sum_{S_{M}} \frac{(-\beta)^{n}(M-n) !}{M !}\left\langle\alpha\left|\prod_{p=1}^{M} \mathcal{H}_{a_{p}, b_{p}}\right| \alpha\right\rangle,
$$

where $a_{p}=1,2$ indicates diagonal $\mathcal{H}_{1, b}=J \Delta S_{i(b)}^{z} S_{j(b)}^{z}+$ $C$ or off-diagonal $\mathcal{H}_{2, b}=J\left(S_{i(b)}^{+} S_{j(b)}^{-}+\right.$h.c. $) / 2$ operators on bond $b$. The constant $C$ is chosen such that all diagonal weights are positive [112], $|\alpha\rangle=\left|S_{1}^{z}, \ldots, S_{L}^{z}\right\rangle$ refers to the $S^{z}$ basis, and $S_{M}=\left[a_{1}, b_{1}\right]\left[a_{2}, b_{2}\right] \ldots\left[a_{M}, b_{M}\right]$ is an index for the operator string $\prod_{p=1}^{M} \mathcal{H}_{a_{p}, b_{p}}$. This string is Metropolis sampled, using two types of updates, (i) diagonal updates which change the number of diagonal operators $\mathcal{H}_{1, b_{p}}$ in the operator string and (ii) loop up- 


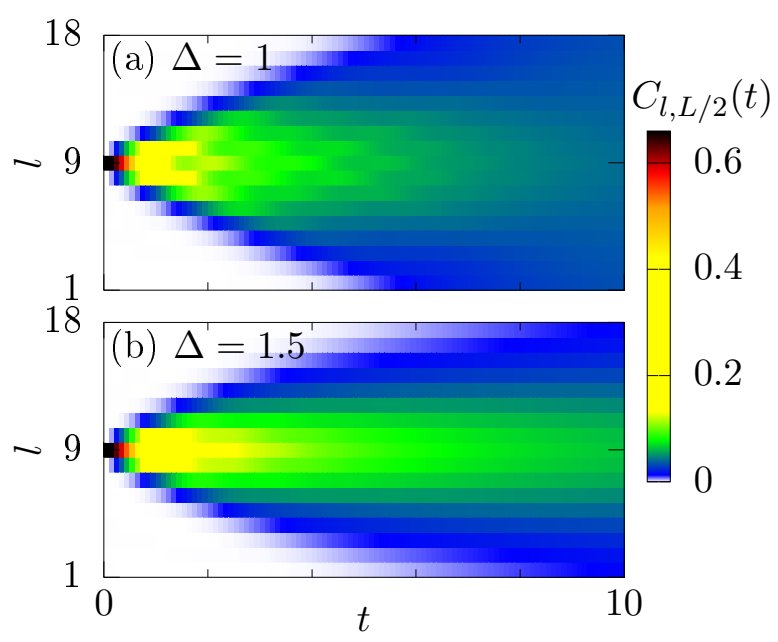

FIG. 3. (Color online) Broadening of density profiles $C_{l, L / 2}(t)$ at infinite temperature $\beta=0$ for (a) $\Delta=1$ and (b) $\Delta=1.5$. We have $L=18$ in both cases.

dates which change the type of operators $\mathcal{H}_{1, b_{p}} \leftrightarrow \mathcal{H}_{2, b_{p}}$. For bipartite lattices the loop update comprises an even number of off-diagonal operators $\mathcal{H}_{2, b_{p}}$, ensuring positivity of the transition probabilities.

From QMC simulations, the real-space correlations $C_{l, l^{\prime}}(\tau)$ are obtained in imaginary time $\tau$,

$$
\begin{array}{r}
C_{l, l^{\prime}}(\tau)=\left\langle\sum_{m=0}^{M}\left(\begin{array}{c}
M \\
m
\end{array}\right)\left(\frac{\tau}{\beta}\right)^{m}\left(1-\frac{\tau}{\beta}\right)^{M-m}\right. \\
\left.\frac{1}{M} \sum_{p=0}^{M-1} S_{l}^{z}(m+p) S_{l^{\prime}}^{z}(p)\right\rangle_{W},
\end{array}
$$

where the argument of $S_{l}^{z}(p)$ refers to discrete expansion slices of the SSE (for details see, e.g., [61]), and $\langle\bullet\rangle_{W}$ denotes the Metropolis weight of an operator string of length $M$ generated by the SSE of $\mathcal{Z}[111,112]$.

After a Fourier transform to momentum space, cf. Eq. (15), the dynamical structure factor eventually results from analytic continuation to real frequencies based on the inversion of

$$
C_{q}(\tau)=\int_{0}^{\infty} d \omega C_{q}(\omega) K(\omega, \tau)
$$

with a kernel $K(\omega, \tau)=\left(e^{-\tau \omega}+e^{-(\beta-\tau) \omega}\right) / \pi$. This inversion is an ill-posed problem, for which maximum entropy methods (MEM) have proven to be well suited. We have applied Bryan's algorithm for our MEM $[113,114]$. This method minimizes the functional $Q=\chi^{2} / 2-\alpha \sigma$, with $\chi$ being the covariance of the QMC data with respect to the MEM trial spectrum $C_{q}(\omega)$. Overfitting is prevented by an entropy term $\sigma=\sum_{\omega} C_{q}(\omega) \ln \left[C_{q}(\omega) / m(\omega)\right]$. We have used a flat default model $m(\omega)$, which is iteratively adjusted to match the zeroth moment of the trial spectrum. The optimal spectrum follows from the weighted average of $C_{q}(\omega)$ with the probability distribution $P\left[\alpha \mid C_{q}(\omega)\right][113]$.

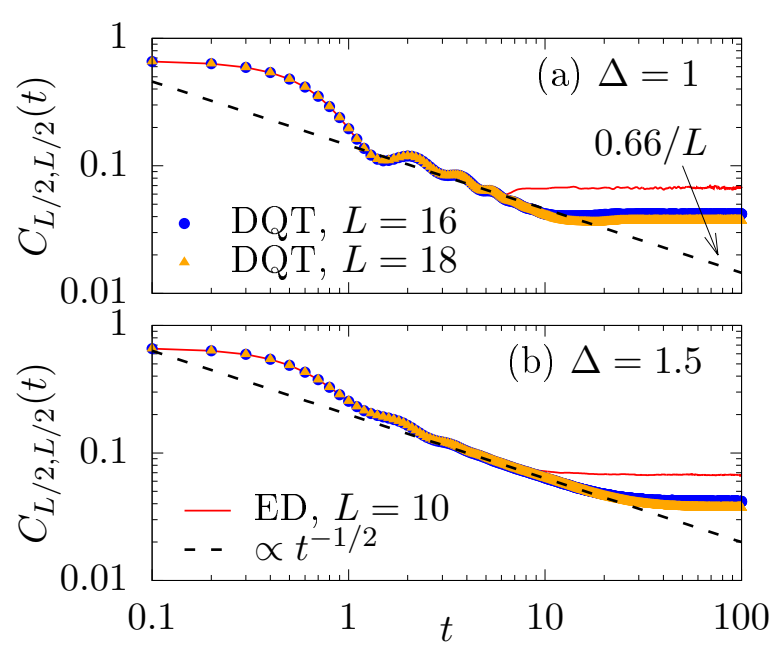

FIG. 4. (Color online) (a) Equal-site correlation $C_{L / 2, L / 2}(t)$ for $\Delta=1$ and system sizes $L=10$ (ED), $L=16,18$ (DQT), in a logarithmic plot. The dashed line indicates power-law decay $\propto 1 / \sqrt{t}$. The constant long-time value scales as $\frac{2}{3} / L$ $[117,118]$. (b) Same data as in (a), but now for the larger anisotropy $\Delta=1.5$. We have $\beta=0$ in both cases.

\section{RESULTS}

We now present our numerical results. First, we study dynamics for infinite temperatures $\beta=0$ and vanishing disorder $W=0$ in Sec. V A. Then, we also consider finite temperatures $\beta>0$ in Sec. VB, before discussing the effect of disorder $W>0$ in Sec. V C.

\section{A. Clean model at high temperatures}

\section{Current dynamics}

Let us start with the discussion of current dynamics for the isotropic model with $\Delta=1$. In Fig. 1 (a), the current autocorrelation function $\langle j(t) j\rangle / L$ is shown for different system sizes $L=12,14,16,18$ in a semilogarithmic plot. First of all, for the small system with $L=12$, we find that the data obtained by DQT reproduce ED results very accurately [76]. As explained in Sec. IV A, this accuracy is expected to improve even further if $L$ is increased, such that the pure-state approach can be regarded as practically exact for all $L \geq 12$. Moreover, while the curves are converged in system size at short times, finite-size effects become apparent for $t \gtrsim 10$. For such times, one finds that $\langle j(t) j\rangle / L$ decays to smaller and smaller values for increasing $L$ (although it is difficult to estimate the $L \rightarrow \infty$ value based on the system sizes numerically available).

Next, Fig. 1 (b) shows the corresponding diffusion coefficient $D(t)$, i.e., essentially the integral over the curves shown in panel (a). Even for the largest system size $L=18$, we observe that $D(t)$ still exhibits a finite slope 

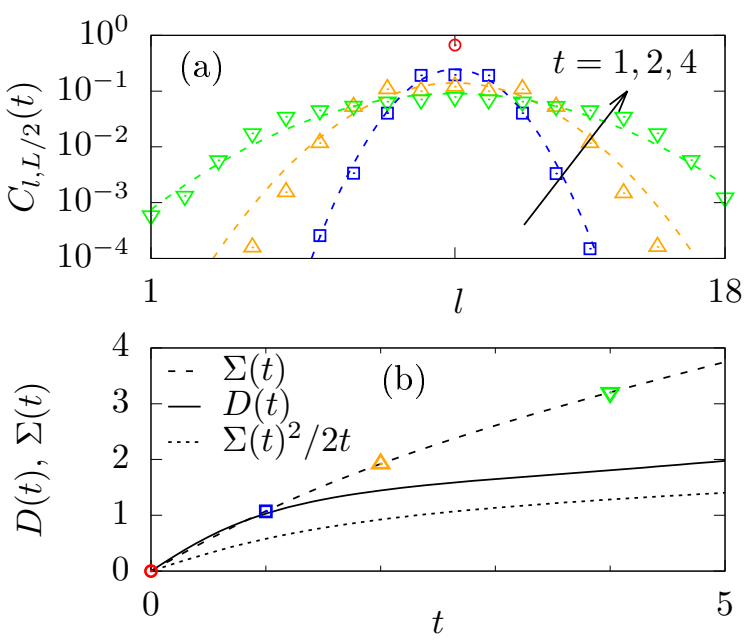

FIG. 5. (Color online) (a) Density profile $C_{l, L / 2}(t)$ at fixed times $t=0,1,2,4$. Dashed curves are Gaussian fits to the data. (b) The width $\Sigma(t)$, as obtained from these density profiles [symbols, Eq. (12)] is compared to the width $\Sigma(t)$, as obtained from current autocorrelations [curve, Eq. (13)]. The derivative $D(t)$ in Eq. (13) as well as the quantity $\Sigma^{2} /(2 t)$ are shown as well. The other parameters are $\Delta=1, L=18$, and $\beta=0$.

$\partial_{t} D(t)>0$ and does not saturate to a constant plateau on the time scales shown here. It is instructive to compare these results to a calculation restricted to the symmetry subspace with $S^{z}=0$. For this choice, one finds that $\langle j(t) j\rangle$ decays significantly faster, cf. Fig. 1 (a), and correspondingly, the diffusion coefficient $D(t)$ is approximately constant for times $t \gtrsim 10$, cf. Fig. 1 (b). Importantly however, the $S^{z}=0$ data in Figs. 1 (a), (b) are converged in system size only up to short times $t \lesssim 5$. Moreover, the convergence towards $L \rightarrow \infty$ is generally slower than a calculation in the full Hilbert space (cf. Ref. [115]), which can also be seen by comparing to results obtained by the time-dependent density matrix renormalization group (tDMRG) [81, 116].

Eventually, Fig. 1 (c) shows the frequency-dependent conductivity $\sigma(\omega)$ for the largest system size $L=18$. We depict data for different frequency resolutions $\delta \omega=\pi / 10$ and $\delta \omega=\pi / 20$, i.e., two rather short cutoff times $t_{\max }$ for the Fourier transform (6). However, even for the short $t_{\text {max }}$ chosen, we observe that $\sigma_{\mathrm{dc}}$ strongly depends on $\delta \omega$ and diverges if $t_{\max }$ is increased. Again, let us compare these results to a calculation in the $S^{z}=0$ subsector only. In this case, the maximum of $\sigma(\omega)$ is shifted to a finite frequency $\omega_{\max } \approx 0.1$ and $\sigma(\omega)$ develops a local minimum at $\omega=0$. Moreover, our DQT results are in good agreement with earlier data obtained by the microcanonical Lanczos method (MCLM) for $S^{z}=0$ [60].

Let us now consider a larger anisotropy $\Delta=1.5$. Analogous to Fig. 1, we present a finite-size scaling of $\langle j(t) j\rangle$ and $D(t)$ in Figs. $2(\mathrm{a}),(\mathrm{b})$. Compared to the isotropic point, we find that $\langle j(t) j\rangle$ now decays to significantly smaller values. Moreover, as can be seen in Fig. 2 (b), the
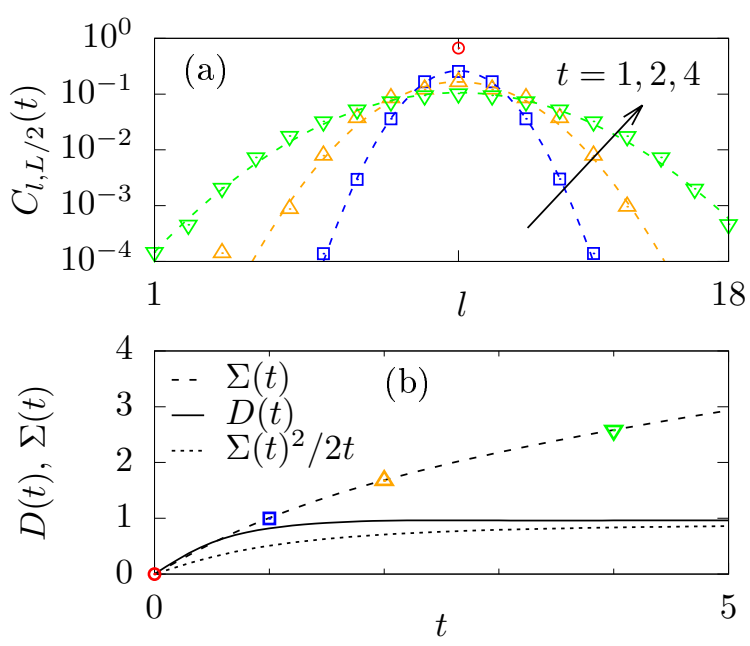

FIG. 6. (Color online) Analogous data as in Fig. 5, but now for the larger anisotropy $\Delta=1.5$. The density profiles in (a) are well described by Gaussians over three orders of magnitude.

diffusion coefficient $D(t)$ converges to an approximately constant and $L$-independent plateau for times $2 \lesssim t \lesssim 5$. In particular, this plateau persists for longer and longer times if $L$ is increased [115].

For system size $L=16$ and $L=18$, we again depict in Fig. 2 (c) the corresponding conductivity $\sigma(\omega)$ for two different frequency resolutions $\delta \omega=\pi / 30$ and $\delta \omega=\pi / 50$. In contrast to $\Delta=1$, cf. Fig. 1 (c), we now find a well-defined dc conductivity $\sigma_{\mathrm{dc}}$, which is practically independent of the specific $L$ and $\delta \omega$ chosen.

Comparing the results presented in Figs. 1 and 2, the dynamics of the spin current apparently exhibits qualitative differences between $\Delta=1$ and $\Delta=1.5$. On the one hand, for the isotropic model, there is a large discrepancy between calculations in the canonical and grandcanonical ensemble. On the other hand, for $\Delta=1.5$, the finite-size scaling of $D(t)$ clearly suggests diffusive spin transport for this value of anisotropy. This is a first central result of the present paper.

\section{Density dynamics}

We now come to the discussion of the spatio-temporal correlation functions $C_{l, l^{\prime}}(t)$. In particular, we here prepare the typical pure state $|\tilde{\psi}(t)\rangle$ by applying the operator $\left(S_{L / 2}^{z}+1\right)^{1 / 2}$, such that the expectation value of $S_{l}^{z}$ yields the correlation $C_{l, L / 2}(t)=\left\langle S_{l}^{z}(t) S_{L / 2}^{z}\right\rangle$, cf. Eqs. (22) and (23). (Note that the specific value $l^{\prime}=L / 2$ is arbitrary due to periodic boundary conditions.)

In Fig. 3, $C_{l, L / 2}(t)$ is shown for $\Delta=1,1.5$ and $L=18$ sites. As discussed in the context of Eq. (10), $C_{l, L / 2}(t)$ exhibits an initial $\delta$-peak profile at $l=L / 2$ which becomes broader for times $t>0$. Moreover, comparing $\Delta=1$ and $\Delta=1.5$, this broadening turns out to be 

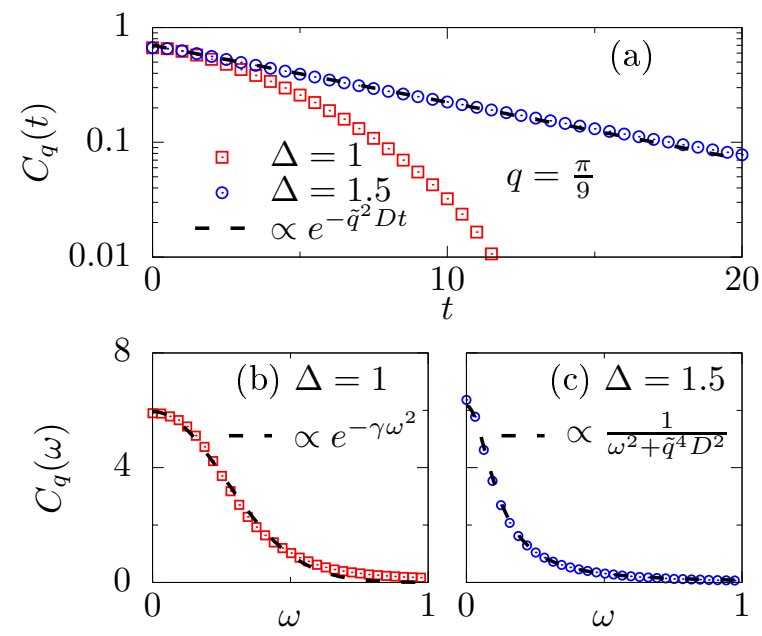

FIG. 7. (Color online) Structure factors for the smallest nonzero momentum $q=\pi / 9$ in a chain with $L=18$. (a) $C_{q}(t)$ for $\Delta=1,1.5$ in a semilogarithmic plot. (b) $C_{q}(\omega)$ for $\Delta=1$. (c) $C_{q}(\omega)$ for $\Delta=1.5$. The dashed lines indicate an exponential decay, as well as Gaussian or Lorentzian line shapes, respectively. We have $\beta=0$ in all cases, and $\delta \omega=\pi / 50$ in (b) and (c).

slower in the case of the larger anisotropy.

Next, let us study the decay of the central peak at $l=L / 2$, i.e., the dynamics of the equal-site correlation function $C_{L / 2, L / 2}(t)$. In Fig. 4, $C_{L / 2, L / 2}(t)$ is shown for $\Delta=1,1.5$ in a logarithmic plot for different system sizes $L=10(\mathrm{ED})$ and $L=16,18$ (DQT). In all cases, we find that $C_{L / 2, L / 2}(t)$ exhibits a fast decay for short times $t \lesssim 1$, followed by a slower decay for $t \lesssim 10$. In particular, for this intermediate regime, $C_{L / 2, L / 2}(t)$ is convincingly described by a power-law $\propto t^{-1 / 2}$, consistent with diffusion phenomenology, cf. Eq. (14). However, comparing $\Delta=1$ and $\Delta=1.5$, it appears that this power-law decay is cleaner for larger $\Delta$. (See also [63] for further data at $\Delta=1$ and temperatures $\beta \geq 0$.) Eventually, for even longer times $t \gtrsim 10, C_{L / 2, L / 2}(t)$ saturates at a constant plateau which is related to the conservation of total magnetization (the plateau scales as $\propto 1 / L$ ), cf. Refs. [117, 118].

In order to analyze the difference between $\Delta=1$ and $\Delta=1.5$ in more detail, Figs. 5 (a) and 6 (a) show cuts of $C_{l, L / 2}(t)$ at fixed times $t=0,1,2,4$ for both values of $\Delta$. In the case of $\Delta=1$ [Fig. 5 (a)], we find that the density profiles exhibit a flat region in the center of the chain which cannot be captured by Gaussian fits. Moreover, as shown in Fig. 5 (b), while the widths $\Sigma(t)$ of these profiles necessarily agree with a calculation via current autocorrelations, cf. Eq. (13), the nonconstant $D(t)$ is inconsistent with diffusion. (As a consequence, $\Sigma(t) \propto t^{\alpha}$ with $\alpha>1 / 2$ and $\Sigma(t)^{2} /(2 t)$ is nonconstant.) In contrast, for $\Delta=1.5$ [Fig. 6 (a)], we find that $C_{l, L / 2}(t)$ is well described by Gaussians over roughly three orders of magnitude for all times shown here. These Gaussian pro-

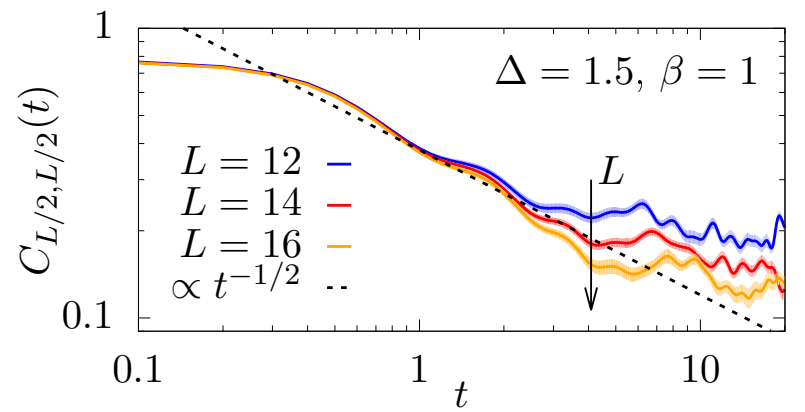

FIG. 8. (Color online) Equal-site correlation $C_{L / 2, L / 2}(t)$ for $\Delta=1.5$ and $L=12,14,16$ at the finite temperature $\beta=1$. Data are averaged over $N_{S}=50$ random initial states and the shaded area indicates the standard deviation of the mean. The dashed line shows the prediction $\propto 1 / \sqrt{t}$ from diffusion phenomenology.

files, in combination with the constant plateau of $D(t)$, the corresponding square-root growth of $\Sigma(t)$, and the saturation of $\Sigma(t)^{2} /(2 t)$ in Fig. 6 (b), are clear signatures of diffusion for this anisotropy. This is another important result of the present paper. Note that a very similar behavior, both for $\Delta=1$ and $\Delta=1.5$, has been found also for $S=1 / 2[23,42]$.

Next, we consider correlations in momentum space. In Fig. 7 (a), the intermediate structure factor $C_{q}(t)$ is shown in a semilogarithmic plot for a single system size $L=18$ and the smallest nonzero momentum $q=\pi / 9$ available. On the one hand, for $\Delta=1.5$, we find that $C_{q}(t)$ exhibits a clean exponential decay with the decay rate $-\tilde{q}^{2} D$, cf. Eq. (17). In particular, let us stress that the dashed line in Fig. 7 (a) is no fit, but takes into account the actual value of $q$ and the diffusion coefficient $D \approx 0.95$ (cf. Refs. [76, 119]), as extracted from the constant plateau in Fig. 2 (b). On the other hand, for $\Delta=1$, $C_{q}(t)$ decays rather quickly and we are unable to detect an exponentially decaying mode for the $q$ values available. This difference between the two anisotropies also carries over to the frequency domain. In Figs. 7 (b), (c) the dynamical structure factor $C_{q=\pi / 9}(\omega)$ is shown for $\Delta=1$ and $\Delta=1.5$, respectively. While for $\Delta=1$, $C_{q}(\omega)$ is very similar to a Gaussian, we observe a pronounced Lorentzian line shape in the case of $\Delta=1.5$, as expected for a diffusive process [cf. Eq. (19)].

\section{Intermediate summary}

Based on the numerical evidence presented in Figs. 1 - 7, high-temperature spin dynamics in the $S=1 \mathrm{XXZ}$ chain appears to be strongly dependent on the value of anisotropy. On the one hand, for $\Delta=1.5$ numerous signatures of genuine spin diffusion can be observed. On the other hand, for $\Delta=1$, these signatures are either less pronounced or entirely absent. While our numerical results cannot rule out that diffusion will eventually 


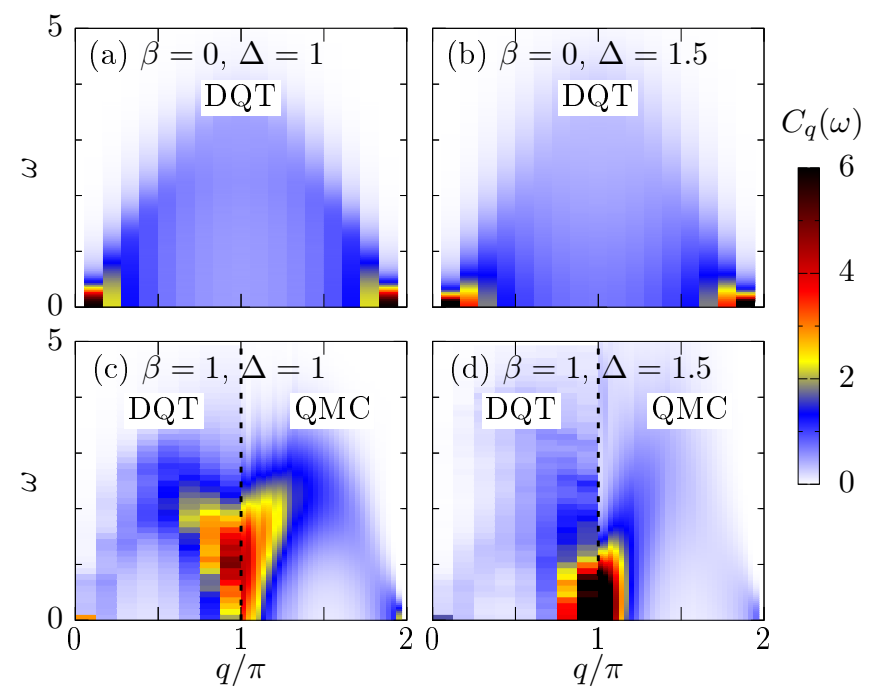

FIG. 9. (Color online) (a), (b) Dynamical structure factor $C_{q}(\omega)$ at infinite temperature $\beta=0$ for $\Delta=1$ and $\Delta=1.5$, obtained by DQT for system size $L=18$. (c), (d) $C_{q}(\omega)$ at $\beta=1$. Data obtained by DQT $\left(N_{S}=50\right)$ for $L=16$ and $q<\pi$ are compared to QMC simulations for $L=64$ at $q>\pi$.

emerge also for $\Delta=1$ asymptotically at long times and larger $L$, they might suggest that high-temperature spin transport in the isotropic $S=1$ Heisenberg chain is superdiffusive, analogous to the case of $S=1 / 2[24,79,80]$, and consistent with recent results in Ref. [81].

\section{B. Clean model at lower temperatures}

In Sec. VA, we have unveiled clear signatures of high-temperature spin diffusion in the easy-axis regime $\Delta=1.5$. Focusing on density dynamics, let us study if such signatures can be found for finite temperatures $\beta>0$ as well. (See also Refs. $[75,77,78,120]$ for transport studies low $T$.)

Figure 8 shows the equal-site correlation $C_{L / 2, L / 2}(t)$ for anisotropic chains with $\Delta=1.5$ and $L=12,14,16$ at the moderate temperature $\beta=1$. The data are averaged over $N_{S}=50$ random initial states in order to account for the larger statistical error of the typicality approximation at $\beta>0$, cf. Sec. IV C, and the shaded area indicates the standard deviation of the mean. Remarkably, we are able to detect an intermediate time window $1 \lesssim t \lesssim 5$, where the decay of $C_{L / 2, L / 2}(t)$ is approximately described by $\propto t^{-1 / 2}$. Even though this scaling is certainly less convincing compared to the infinite-temperature case shown in Fig. 4 (b), it suggests that diffusion might occur also at finite temperatures $T \sim J$.

Next, Fig. 9 shows a contour plot of the dynamical structure factor $C_{q}(\omega)$ for all four possible combinations of $\beta=0,1$ and $\Delta=1,1.5$. On the one hand, for $\beta=0$ [Figs. 9 (a), (b)], the data are obtained by means of DQT for chains with $L=18$. We find that $C_{q}(\omega)$ exhibits a
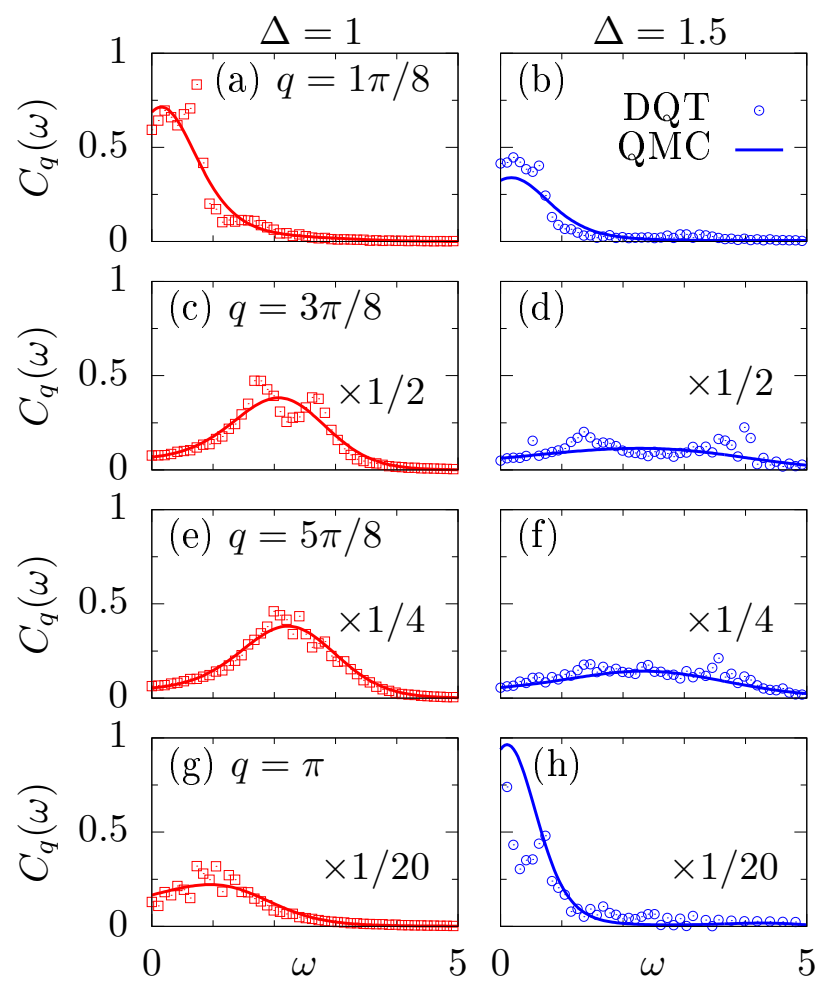

FIG. 10. (Color online) Dynamical structure factor $C_{q}(\omega)$ at finite temperature $\beta=1$ and various momenta $q$, both for $\Delta=1$ (left) and $\Delta=1.5$ (right). The data are obtained by DQT (symbols) and QMC (curves) for chains with $L=16$. Note that the data in panels (c) - (h) have been multiplied by a factor for better visibility.

broad excitation continuum in the center of the Brillouin zone extending up to $\omega \lesssim 5$, as well as distinct (diffusion) poles for small wave numbers $q \rightarrow 0$ [which have been discussed in detail in the context of Figs. 7 (b), (c)]. On the other hand, for $\beta=1$ [Figs. 9 (c), (d)], we compare data obtained by DQT for $L=16$ to QMC simulations for significantly larger systems with $L=64$ sites. One clearly observes that the lowering of the temperature leads to a redistribution of spectral weight. Specifically, we find increased intensity around $q=\pi$, which is most pronounced for $\Delta=1.5$. Correspondingly, the spectral weight of the original poles for momenta $q \rightarrow 0$ is reduced at $\beta=1$. Moreover, considering the big difference in system size, the agreement between DQT and QMC is quite convincing. For a thorough discussion of $C_{q}(\omega)$ at $\Delta=1$ and lower temperatures $T \ll J$ see, e.g., Refs. $[61,62]$.

For a more detailed comparison between DQT and QMC as well as between $\Delta=1$ and $\Delta=1.5$, we depict cuts of $C_{q}(\omega)$ at $\beta=1$ for various momenta $q$ in Figs. 10 (a) - (h). In particular, DQT and QMC data are compared for the same chain length $L=16$. For all combinations of $q$ and $\Delta$ shown here, we find that DQT and QMC agree very well. While the DQT data are somewhat noisy due to the finite chain length, the 


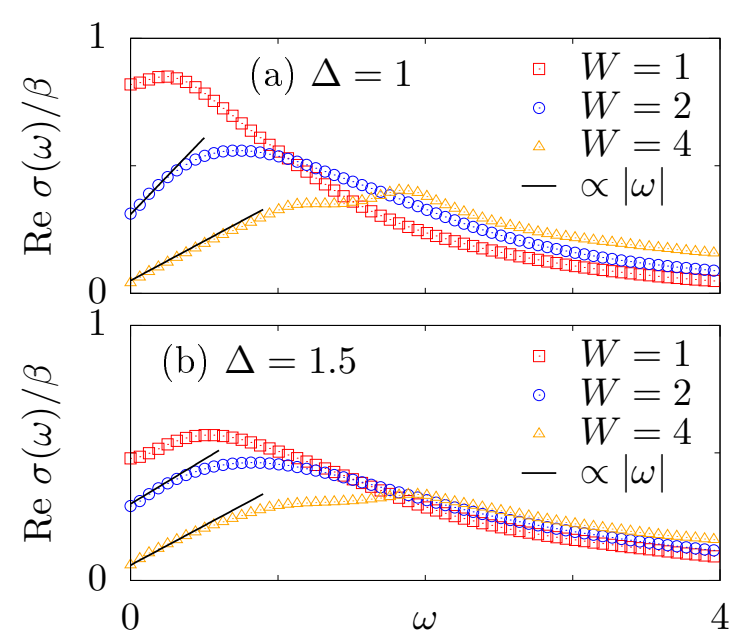

FIG. 11. (Color online) Conductivity $\sigma(\omega)$ for disorder $W=$ $1,2,4$ and anisotropies (a) $\Delta=1$, (b) $\Delta=1.5$. In the low$\omega$ regime, the conductivity is well described by power laws, $\operatorname{Re} \sigma(\omega) \approx \sigma_{\mathrm{dc}}+a|\omega|^{\alpha}$, with $\alpha=1$, cf. Refs. [123, 124]. We have $\beta=0, L=16$, and $N=100$ in all cases.

QMC curves are naturally very smooth. Moreover, due to difficulties within the analytic continuation, it is hard to resolve certain fine structure of $C_{q}(\omega)$ in QMC simulations such as, e.g., the double peak in Fig. 10 (c) (see also the discussion in [62]).

For the smallest momentum $q=\pi / 8$ available [Figs. 10 (a), (b)], we find that $C_{q}(\omega)$ behaves qualitatively similar for $\Delta=1$ and $\Delta=1.5$. Specifically, in both cases $C_{q}(\omega)$ has a pole at $\omega \approx 0$, reminiscent of the $\beta=$ 0 results discussed in Figs. 7 (b), (c). Moreover, the maximum of $C_{q}(\omega)$ seems to be slightly shifted to finite frequencies $\omega>0$, although this can be a finite-size effect. Next, for momenta $q \approx \pi / 2$ [Figs. 10 (c)-(f)], we find that $C_{q}(\omega)$ exhibits a distinct excitation mode in the isotropic case, whereas the spectrum for $\Delta=1.5$ is rather flat. Furthermore, as shown in Figs. $10(\mathrm{~g}),(\mathrm{h})$, there is high spectral weight at $q=\pi$, and $C_{q}(\omega)$ has a pronounced peak at $\omega \approx 0$ for $\Delta=1.5$ (consistent with a Néel phase for this $\Delta$ at low $T$ [121]).

\section{Disordered model at high temperatures}

Eventually, let us study the effect of disorder on the spin dynamics, focusing on high temperatures $\beta=0$. Due to the additional numerical costs caused by the necessity to average over different disorder realizations, we here restrict ourselves to a maximum system size of $L=16$.

Analogous to Sec. VA, we start our discussion with current dynamics. In Fig. 11, the conductivity $\sigma(\omega)$ is shown for $\Delta=1,1.5$ and various values of disorder $W=1,2,4$. Overall, we find a very similar behavior for both values of the exchange anisotropy. Specifically, for

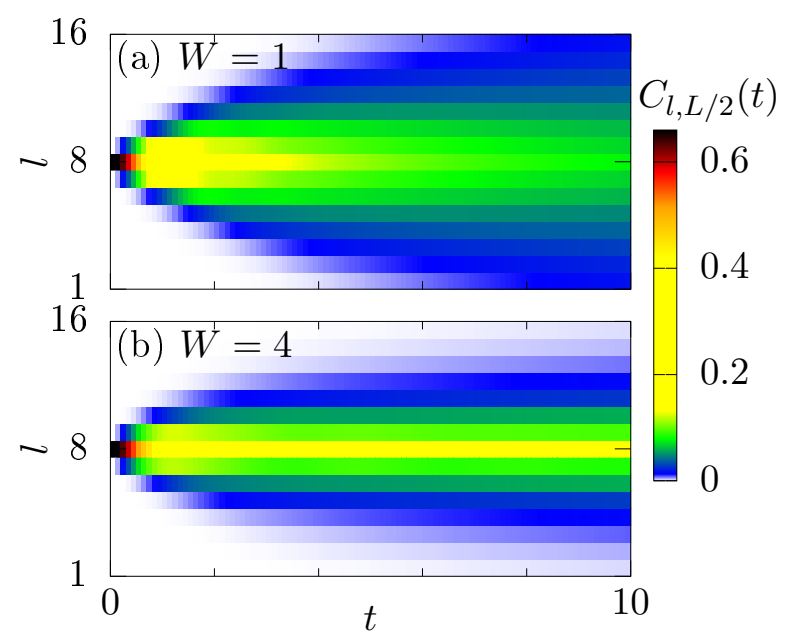

FIG. 12. (Color online) Broadening of density profiles $C_{l, L / 2}(t)$ for different values of disorder (a) $W=1$, (b) $W=4$. We have $\Delta=1.5, \beta=0, L=16$, and $N=100$ in both cases.

all cases shown here, one observes that $\sigma(\omega)$ has a welldefined dc conductivity $\sigma_{\mathrm{dc}}$, which decreases for larger $W$. Moreover, one finds that the maximum of the conductivity $\sigma_{\max }>\sigma_{\mathrm{dc}}$ is shifted to larger and larger $\omega$ if disorder is increased. Furthermore, for $W=2,4$ and low frequencies $\omega \lesssim 1$, the conductivity is well described by a power law, $\operatorname{Re} \sigma(\omega) \approx \sigma_{\mathrm{dc}}+a|\omega|^{\alpha}$, with $\alpha=1[87,122]$. Note that qualitatively similar results for $S=1 / 2$ can be found in Refs. [123, 124].

Next, let us also discuss the dynamics of spatiotemporal correlations $C_{l, L / 2}(t)$ in the presence of disorder. We here particularly focus on the easy-axis regime $\Delta=1.5$. For this value of $\Delta$, we have unveiled various signatures of diffusion in the disorder-free case $W=0$, cf. Figs. 3 - 7. These data now serve as a benchmark for the study of $W>0$. In Figs. 12 (a) and (b), contour plots of $C_{l, L / 2}(t)$ are shown for $W=1$ and $W=4$, respectively. Analogous to Fig. 3, $C_{l, L / 2}(t)$ initially exhibits a $\delta$ peak at $t=0$, which broadens for times $t>0$. However, this broadening becomes slower if $W$ is increased, with more weight remaining close to the center of the chain.

For a more detailed analysis, Fig. 13 shows cuts of $C_{l, L / 2}(t)$ at fixed times $t=1,5,10$ for both, weak disorder $W=1[(\mathrm{a})$ - (c)] and stronger disorder $W=4[(\mathrm{~d})$ (f)]. On the one hand, for $W=1$, we find that $C_{l, L / 2}(t)$ is again in good agreement with a Gaussian profile at short times $t=1$. In comparison with the $W=0$ data shown in Fig. 6 (a), this agreement becomes slightly less convincing for later times $t=5$ [Fig. 13 (b)]. On the other hand, for $W=4, C_{l, L / 2}(t)$ exhibits a completely different behavior. For times $t=5$ and 10, cf. Figs. 13 (e), (f), the profiles are not described by Gaussians anymore, but are rather of triangular shape in the semilogarithmic plot used. Such exponentially decaying tails are clearly inconsistent with diffusion and might suggest the presence of a nondiffusive regime $[118,125,126]$. 

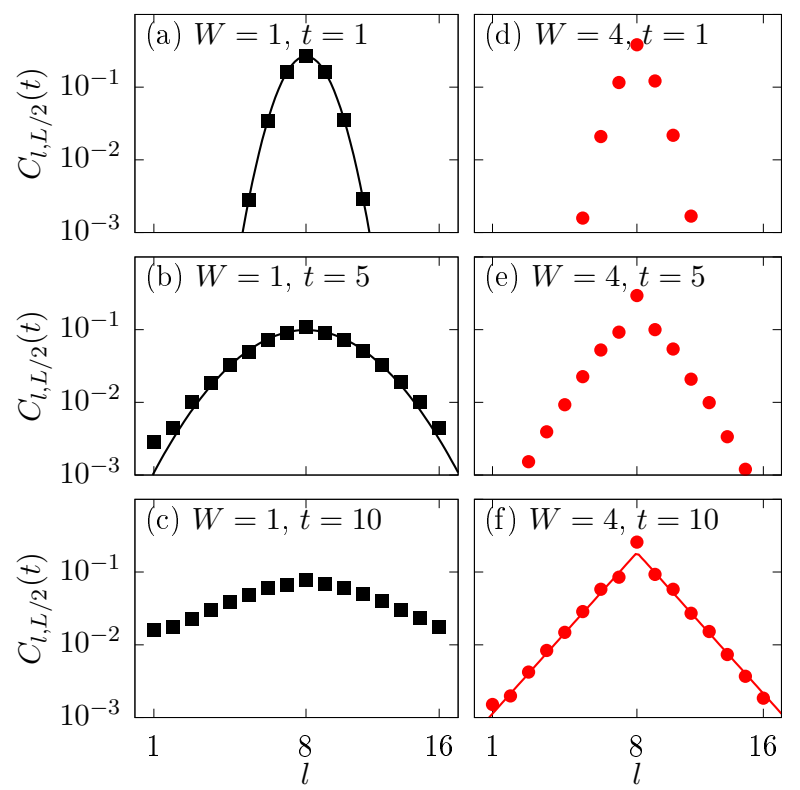

FIG. 13. (Color online) $C_{l, L / 2}(t)$ at fixed times $t=1,5,10$ for $W=1[(\mathrm{a})-(\mathrm{c})]$ and $W=4[(\mathrm{~d})-(\mathrm{f})]$ in a semilogarithmic plot. Curves indicate Gaussian or exponential fits. We have $\Delta=1.5, \beta=0, L=16$, and $N=100$ in all cases.

Furthermore, the equal-site correlation function $C_{L / 2, L / 2}(t)$ is shown in Fig. 14 for $W=0,1,2,4$ in a logarithmic plot. While the curves for $W=0$ and $W=1$ are still very similar to each other, we find that $C_{L / 2, L / 2}(t)$ decays slowly for strong disorder $W=4$ and is inconsistent with $\propto t^{-1 / 2}$. (For a study of the Fourier transform of $C_{L / 2, L / 2}(t)$ in disordered spin-1/2 models, see [127])

Figure 15 (a) shows the intermediate structure factor $C_{q}(t)$ for the smallest nonzero momentum $q=\pi / 8$. For increasing $W$, we find that the slope of $C_{q}(t)$ becomes more and more flat, which can be interpreted as a shrinking of the diffusion constant. In particular, for strong disorder $W=4, C_{q}(t)$ essentially does not decay at all on the time scales depicted. The nondecaying behavior of $C_{q}(t)$ is also reflected in its Fourier transform $C_{q}(\omega)$, which is shown in Figs. 15 (b), (c) in terms of a contour

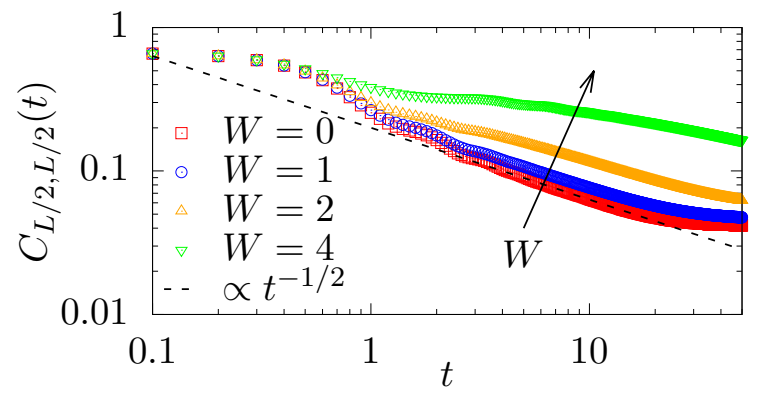

FIG. 14. (Color online) $C_{L / 2, L / 2}(t)$ for disorder $W=0,1,2,4$ in a logarithmic plot. We have $\Delta=1.5, \beta=0, L=16$, and $N=100$ in all cases.
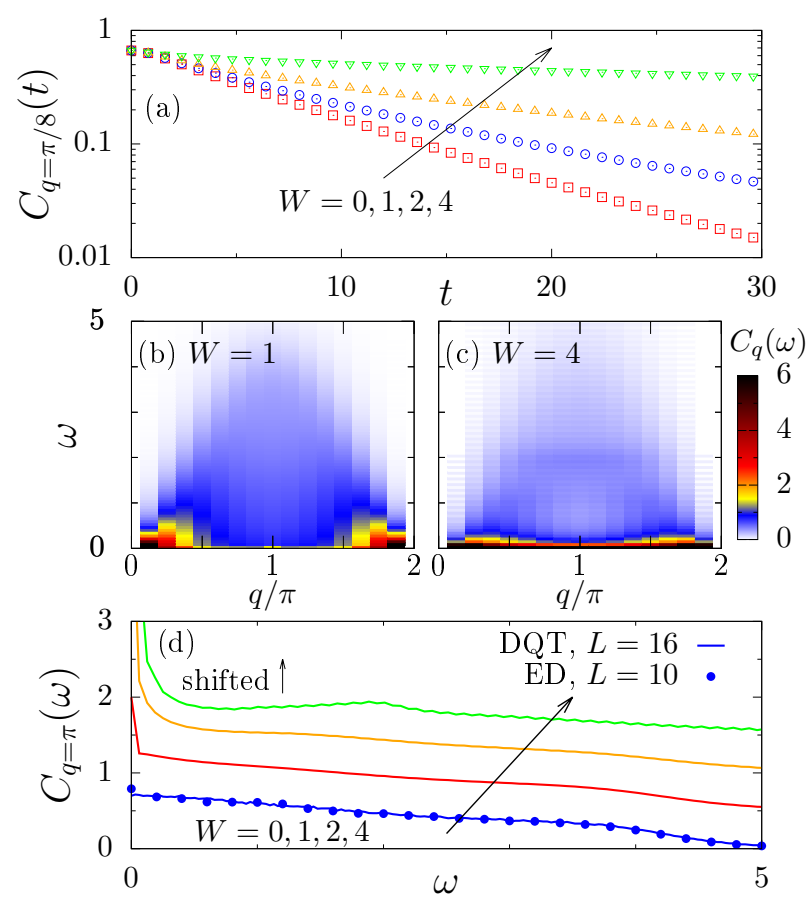

FIG. 15. (Color online) (a) $C_{q}(t)$ at momentum $q=\pi / 8$ for disorder $W=0,1,2,4$ in a semilogarithmic plot. (b), (c) Contour plots of $C_{q}(\omega)$ for disorder $W=1$ and $W=4$, respectively. (d) $C_{q}(\omega)$ at momentum $q=\pi$ for disorder $W=0,1,2,4$. For $W=0$, we additionally compare to $\mathrm{ED}$ $(L=10)$. The curves for $W>0$ are shifted by constant offsets in order to improve the visibility. We have $\Delta=1.5$, $\beta=0, L=16$, and $N=100$ in all cases.

plot for $W=1$ and $W=4$, respectively. While $C_{q}(\omega)$ still exhibits a broad excitation continuum in the center of the Brillouin zone [cf. Fig. 9 (b) for $W=0$ ], we find that $C_{q}(\omega)$ additionally develops a high contribution at $\omega=0$ if $W$ is increased. On the one hand, for $W=1$, this peaked structure is pronounced for $q \rightarrow 0$, e.g., due to diffusion. On the other hand, for strong disorder $W=4$, the high contribution in $C_{q}(\omega)$ can be clearly identified for all momenta $q$ in the Brillouin zone. Note that a very similar behavior has also been observed in the case of spin $S=1 / 2$ [124]. To illustrate the development of this high contribution, Fig. $15(\mathrm{~d})$ shows $C_{q}(\omega)$ for $W=0,1,2,4$ at the fixed momentum $q=\pi$. Note that the data for different values of $W$ are artificially shifted in the vertical direction to improve visibility. For all values of disorder shown here, we find that $C_{q=\pi}(\omega)$ has an almost featureless shape for finite $\omega$ and roughly extends up to $\omega \lesssim 5$. However, one can clearly observe that the high contribution at $\omega \approx 0$ becomes more pronounced for increasing $W$.

The numerical data presented in Figs. 11 - 15 suggest that the spin-1 XXZ chain undergoes a transition between a diffusive regime and a nondiffusive phase for sufficiently strong disorder. 


\section{CONCLUSION}

To summarize, we have studied the magnetization dynamics in the one-dimensional $S=1$ XXZ model for various anisotropies and temperatures, as well as in the presence of quenched disorder induced by a random magnetic field.

As a main result, we unveiled that high-temperature spin transport is diffusive in the easy-axis regime for strong exchange anisotropies. This finding was based on the combination of numerous signatures, such as (i) Gaussian spreading of correlations, (ii) a timeindependent diffusion coefficient, (iii) power-law decay of equal-site correlations, (iv) exponentially decaying longwavelength modes, and (v) Lorentzian line shapes of the dynamical structure factor. Besides, we provided evidence that some of these signatures are not exclusively restricted to the infinite-temperature limit, but can persist at lower temperatures as well. For these lower temperatures, we particularly found a very good agreement between the pure-state typicality approach and additional quantum Monte Carlo simulations.

In contrast, we demonstrated that a proper analysis of magnetization dynamics is considerably more delicate for the isotropic case $\Delta=1$. Specifically, we found that even for the largest system sizes amenable to our numerical approach, the signatures (i) - (v) are either less pronounced or entirely absent. Therefore, our numerical analysis suggests that high-temperature spin transport might be superdiffusive in the $S=1$ Heisenberg chain despite the nonintegrability of the model. This finding is consistent with recent results in Ref. [81].

Eventually, upon introducing a random on-site magnetic field, we observed a breakdown of diffusion and distinctly slower dynamics. Moreover, our results exhibit qualitative similarities to disordered spin- $1 / 2$ chains and might be consistent with the presence of a nondiffusive regime.

Promising directions of research include, e.g., the application of the pure-state approach to spin dynamics for $S \geq 1$ at finite and infinite temperature. In particu- lar, a more detailed analysis of a putative transition to a many-body localized phase in models with $S \geq 1$ is an interesting avenue of future work.

\section{Acknowledgements}

This work has been funded by the Deutsche Forschungsgemeinschaft (DFG) - Grants No. 397067869 (STE 2243/3-1), No. 355031190 - within the DFG Research Unit FOR 2692. N. C. and W. B. acknowledge financial support from "Niedersächsisches Vorab" through "Quantum- and Nano-Metrology (QUANOMET)" initiative within the project NP-2.

\section{Appendix A: Typicality relation}

Let us briefly derive the typicality relation given in Eq. (22) of the main text. To this end, we start with a correlation function at formally infinite temperature,

$$
\begin{aligned}
\frac{\operatorname{Tr}\left[S_{l}^{z}(t)\left(S_{l^{\prime}}^{z}+1\right)\right]}{d} & =\frac{\operatorname{Tr}\left[S_{l}^{z}(t) S_{l^{\prime}}^{z}\right]}{d}+\frac{\operatorname{Tr}\left[S_{l}^{z}(t)\right]}{d} \\
& =C_{l, l^{\prime}}(t)
\end{aligned}
$$

where we have used that $\operatorname{Tr}\left[S_{l}^{z}\right]=0$. Thus, the expression $\operatorname{Tr}\left[S_{l}^{z}(t)\left(S_{l^{\prime}}^{z}+1\right)\right] / d$ is equivalent to the correlation function $C_{l, l^{\prime}}(t)$ from Eq. (9). Exploiting this fact, we can now write

$$
\begin{aligned}
\frac{\operatorname{Tr}\left[S_{l}^{z}(t)\left(S_{l^{\prime}}^{z}+1\right)\right]}{d} & =\frac{\operatorname{Tr}\left[\sqrt{S_{l^{\prime}}^{z}+1} S_{l}^{z}(t) \sqrt{S_{l^{\prime}}^{z}+1}\right]}{d} \\
& \approx \frac{\left\langle\varphi\left|\sqrt{S_{l^{\prime}}^{z}+1} S_{l}^{z}(t) \sqrt{S_{l^{\prime}}^{z}+1}\right| \varphi\right\rangle}{\langle\varphi \mid \varphi\rangle} \\
& =\left\langle\tilde{\psi}(t)\left|S_{l}^{z}\right| \tilde{\psi}(t)\right\rangle,
\end{aligned}
$$

where we have used the cyclic invariance of the trace and the definition of the pure state $|\tilde{\psi}(t)\rangle=e^{-i \mathcal{H} t}|\tilde{\psi}(0)\rangle$, cf. Eq. (23). Note that the statistical error $\epsilon$ of the typicality approximation has been dropped for clarity in Eq. (A4).
[1] A. Polkovnikov, K. Sengupta, A. Silva, and M. Vengalattore, Rev. Mod. Phys. 83, 863 (2011).

[2] C. Gogolin and J. Eisert, Rep. Prog. Phys. 79, 056001 (2016).

[3] L. D'Alessio, Y. Kafri, A. Polkovnikov, and M. Rigol, Adv. Phys. 65, 239 (2016).

[4] J. M. Deutsch, Phys. Rev. A 43, 2046 (1991).

[5] M. Srednicki, Phys. Rev. E 50, 888 (1994).

[6] M. Rigol, V. Dunjko, and M. Olshanii, Nature 452, 854 (2008).

[7] U. Schollwöck, Rev. Mod. Phys. 77, 259 (2005); Ann. Phys. 326, 96 (2011).

[8] T. Langen, R. Geiger, and J. Schmiedmayer, Ann. Rev. Condens. Matter Phys. 6, 201 (2015).
[9] R. Blatt and C. F. Roos, Nat. Phys. 8, 277 (2012).

[10] M. Buchanan, Nat. Phys. 1, 71 (2005).

[11] F. H. L. Essler and M. Fagotti, J. Stat. Mech. 2016, 064002 (2016).

[12] L. Vidmar and M. Rigol, J. Stat. Mech. 2016, 064007 (2016).

[13] X. Zotos, F. Naef, and P. Prelovšek, Phys. Rev. B 55, 11029 (1997).

[14] T. Prosen and E. Ilievski, Phys. Rev. Lett. 111, 057203 (2013).

[15] F. Heidrich-Meisner, A. Honecker, D. C. Cabra, and W. Brenig, Phys. Rev. B 68, 134436 (2003).

[16] O. A. Castro-Alvaredo, B. Doyon, and T. Yoshimura, Phys. Rev. X 6, 041065 (2016). 
[17] B. Bertini, M. Collura, J. De Nardis, and M. Fagotti, Phys. Rev. Lett. 117, 207201 (2016).

[18] J. De Nardis, D. Bernard, and B. Doyon, Phys. Rev. Lett. 121, 160603 (2018).

[19] J. Sirker, R. G. Pereira, and I. Affleck, Phys. Rev. B 83, 035115 (2011).

[20] M. Žnidarič, Phys. Rev. Lett. 106, 220601 (2011).

[21] R. Steinigeweg and W. Brenig, Phys. Rev. Lett. 107, 250602 (2011).

[22] C. Karrasch, J. E. Moore, and F. Heidrich-Meisner, Phys. Rev. B 89, 075139 (2014).

[23] R. Steinigeweg, F. Jin, D. Schmidtke, H. de Raedt, K. Michielsen, and J. Gemmer, Phys. Rev. B 95, 035155 (2017).

[24] M. Ljubotina, M. Žnidarič, and T. Prosen, Nat. Comm. 8, 16117 (2017).

[25] T. Prosen and M. Žnidarič, Phys. Rev. B 86, 125118 (2012).

[26] C. Karrasch, T. Prosen, and F. Heidrich-Meisner, Phys. Rev. B 95, 060406(R) (2017).

[27] R. Steinigeweg, F. Jin, H. De Raedt, K. Michielsen, and J. Gemmer, Phys. Rev. E 96, 020105(R) (2017).

[28] A. L. Chernyshev and A. V. Rozhkov, Phys. Rev. Lett. 116, 017204 (2016)

[29] D. Jansen, J. Stolpp, L. Vidmar, and F. HeidrichMeisner, Phys. Rev. B 99, 155130 (2019).

[30] K. R. Hazzard, M. van den Worm, M. Foss-Feig, S. R. Manmana, E. G. Dalla Torre, T. Pfau, M. Kastner, and A. M. Rey, Phys. Rev. A 90, 063622 (2014).

[31] B. Kloss and Y. Bar Lev, Phys. Rev. A 99, 032114 (2019).

[32] A. Metavitsiadis, X. Zotos, O. S. Barišić, and P. Prelovšek, Phys. Rev. B 81, 205101 (2010).

[33] M. Brenes, E. Mascarenhas, M. Rigol, and J. Goold, Phys. Rev. B 98, 235128 (2018).

[34] J. Herbrych, J. Kokalj, and P. Prelovšek, Phys. Rev. Lett. 111, 147203 (2013).

[35] F. Borgonovi, F. M. Izrailev, L. F. Santos, and V. G. Zelevinsky, Phys. Rep. 626, 1 (2016).

[36] C. W. von Keyserlingk, T. Rakovszky, F. Pollmann, and S. L. Sondhi, Phys. Rev. X 8, 021013 (2018).

[37] A. Nahum, S. Vijay, and J. Haah, Phys. Rev. X 8, 021014 (2018).

[38] V. Khemani, A. Vishwanath, and D. A. Huse, Phys. Rev. X 8, 031057 (2018).

[39] M. Michel, G. Mahler, and J. Gemmer, Phys. Rev. Lett. 95, 180602 (2005).

[40] C. Mejía-Monasterio, T. Prosen, and G. Casati, Europhys. Lett. 72, 520 (2005).

[41] M. Medenjak, K. Klobas, and T. Prosen, Phys. Rev. Lett. 119, 110603 (2017).

[42] J. Richter, F. Jin, H. De Raedt, K. Michielsen, J. Gemmer, and R. Steinigeweg, Phys. Rev. B 97, 174430 (2018).

[43] J. Richter, F. Jin, L. Knipschild, J. Herbrych, H. De Raedt, K. Michielsen, J. Gemmer, and R. Steinigeweg, Phys. Rev. B 99, 144422 (2019).

[44] F. D. M. Haldane, Phys. Rev. Lett. 50, 1153 (1983).

[45] H.-J. Mikeska and A. K. Kolezhuk, Lect. Notes Phys. 645, 1 (2004).

[46] S. Lloyd, Ph.D. Thesis, The Rockefeller University (1988), Chapter 3, arXiv:1307.0378.

[47] J. Gemmer, M. Michel, and G. Mahler, Quantum Thermodynamics (Springer, Berlin, 2004).
[48] S. Popescu, A. J. Short, and A. Winter, Nat. Phys. 2 754 (2006).

[49] S. Goldstein, J. L. Lebowitz, R. Tumulka, and N. Zanghì, Phys. Rev. Lett. 96, 050403 (2006).

[50] P. Reimann, Phys. Rev. Lett. 99, 160404 (2007).

[51] A. Hams and H. De Raedt, Phys. Rev. E 62, 4365 (2000).

[52] T. Iitaka and T. Ebisuzaki, Phys. Rev. Lett. 90, 047203 (2003).

[53] S. Sugiura and A. Shimizu, Phys. Rev. Lett. 111, 010401 (2013).

[54] T. A. Elsayed and B. V. Fine, Phys. Rev. Lett. 110, 070404 (2013).

[55] T. Monnai and A. Sugita, J. Phys. Soc. Jpn. 83, 094001 (2014).

[56] R. Steinigeweg, J. Gemmer, and W. Brenig, Phys. Rev. Lett. 112, 120601 (2014).

[57] L. Piroli and E. Vernier, J. Stat. Mech. 2016, 053106 (2016).

[58] J. Deisz, M. Jarrell, and D. L. Cox, Phys. Rev. B 42, 4869(R) (1990).

[59] S. R. White and D. A. Huse, Phys. Rev. B 48, 3844 (1993)

[60] J. Karadamoglou and X. Zotos, Phys. Rev. Lett. 93, 177203 (2004).

[61] S. N. Grossjohann, Static and Dynamic Properties of Low Dimensional Quantum Spin Systems, dissertation, Technische Universität Braunschweig, Cuvillier Verlag Göttingen, 2010.

[62] J. Becker, T. Köhler, A. C. Tiegel, S. R. Manmana, S. Wessel, and A. Honecker, Phys. Rev. B 96, 060403(R) (2017).

[63] S. Capponi, M. Dupont, A. W. Sandvik, and P. Sengupta, Phys. Rev. B 100, 094411 (2019).

[64] L. P. Regnault, I. Zaliznyak, J. P. Renard, and C. Vettier, Phys. Rev. B 50, 9174 (1994).

[65] M. Orendáč, A. Orendáčová, J. Černác, A. Feher, P. J. C. Signore, M. W. Meisel, S. Merah, and M. Verdaguer, Phys. Rev. B 52, 3435 (1995).

[66] Z. Honda, H. Asakawa, and K. Katsumata, Phys. Rev. Lett. 81, 2566 (1998).

[67] M. Kenzelmann, R. A. Cowley, W. J. L. Buyers, Z. Tun, R. Coldea, and M. Enderle, Phys. Rev. B 66, 024407 (2002).

[68] M. Takigawa, T. Asano, Y. Ajiro, M. Mekata, and Y. J. Uemura, Phys. Rev. Lett. 76, 2173 (1996).

[69] A. V. Sologubenko, S. M. Kazakov, H. R. Ott, T. Asano, and Y. Ajiro, Phys. Rev. B 68, 094432 (2003).

[70] I. Affleck, Phys. Rev. B 43, 3215 (1991).

[71] Y. Rahnavard and W. Brenig, Phys. Rev. B 91, 054405 (2015).

[72] S. C. Furuya, T. Suzuki, S. Takayoshi, Y. Maeda, and M. Oshikawa, Phys. Rev. B 84, 180410(R) (2011).

[73] J. Herbrych and X. Zotos, Phys. Rev. B 93, 134412 (2016).

[74] F. Lange, S. Ejima, and H. Fehske, Phys. Rev. B 97, 060403(R) (2018).

[75] S. Sachdev and K. Damle, Phys. Rev. Lett. 78, 943 (1997).

[76] R. Steinigeweg and R. Schnalle, Phys. Rev. E 82, 040103(R) (2010).

[77] S. Fujimoto, J. Phys. Soc. Jpn. 68, 2810 (1999).

[78] R. M. Konik, Phys. Rev. B 68, 104435 (2003). 
[79] S. Gopalakrishnan and R. Vasseur, Phys. Rev. Lett. 122, 127202 (2019).

[80] J. Richter and R. Steinigeweg, Phys. Rev. B 99, 094419 (2019).

[81] J. De Nardis, M. Medenjak, C. Karrasch, E. Ilievski, arXiv:1903.07598.

[82] X. Zotos, Phys. Rev. Lett. 82, 1764 (1999).

[83] A. Urichuk, Y. Oez, A. Klümper, and J. Sirker, SciPost Phys. 6, 005 (2019).

[84] D. M. Basko, I. L. Aleiner, and B. L. Altshuler, Ann. Phys. 321, 1126 (2006).

[85] R. Nandkishore and D. A. Huse, Annu. Rev. Condens. Matter Phys. 6, 15 (2015).

[86] T. C. Berkelbach and D. R. Reichman, Phys. Rev. B 81, 224429 (2010).

[87] S. Gopalakrishnan, M. Müller, V. Khemani, M. Knap, E. Demler, and D. A. Huse, Phys. Rev. B 92, 104202 (2015).

[88] D. J. Luitz, N. Laflorencie, and F. Alet, Phys. Rev. B 91, 081103(R) (2015).

[89] B. Bauer and C. Nayak, J. Stat. Mech. 2013, P09005 (2013)

[90] D. A. Huse, R. Nandkishore, and V. Oganesyan, Phys. Rev. B 90, 174202 (2014).

[91] M. Serbyn, Z. Papić, and D. A. Abanin, Phys. Rev. Lett. 111, 127201 (2013).

[92] M. Žnidarič, T. Prosen, and P. Prelovšek, Phys. Rev. B 77, 064426 (2008).

[93] J. H. Bardarson, F. Pollmann, and J. E. Moore, Phys. Rev. Lett. 109, 017202 (2012).

[94] C. Monthus, O. Golinelli, and Th. Jolicœur Phys. Rev. B 58, 805 (1998).

[95] G. Refael and J. E. Moore, Phys. Rev. B 76, 024419 (2007).

[96] F. Heidrich-Meisner, A. Honecker, and W. Brenig, Eur. Phys. J. Spec. Top. 151, 135 (2007).

[97] R. Steinigeweg and J. Gemmer, Phys. Rev. B 80, 184402 (2009).

[98] R. Steinigeweg, H. Wichterich, and J. Gemmer, Europhys. Lett. 88, 10004 (2009).

[99] Y. Yan, F. Jiang, and H. Zhao, Eur. Phys. J. B 88, 11 (2015).

[100] D. J. Luitz and Y. B. Lev, Ann. Phys. 529, 1600350 (2017).

[101] K. Fabricius, U. Löw, and J. Stolze, Phys. Rev. B 55, 5833 (1997).

[102] R. Steinigeweg, J. Gemmer, and W. Brenig, Phys. Rev. B 91, 104404 (2015).

[103] C. Bartsch and J. Gemmer, Phys. Rev. Lett. 102, 110403 (2009).

[104] J. Richter and R. Steinigeweg, Phys. Rev. E 99, 012114 (2019).
[105] H. De Raedt and K. Michielsen, in Handbook of Theoretical and Computational Nanotechnology (American Scientific Publishers, Los Angeles, 2006).

[106] V. V. Dobrovitski and H. De Raedt, Phys. Rev. E 67, 056702 (2003).

[107] A. Weiße, G. Wellein, A. Alvermann, and H. Fehske, Rev. Mod. Phys. 78, 275 (2006).

[108] V. K. Varma, A. Lerose, F. Pietracaprina, J. Goold, and A. Scardicchio, J. Stat. Mech. 2017053101 (2017).

[109] I. Rousochatzakis, S. Kourtis, J. Knolle, R. Moessner, and N. B. Perkins, Phys. Rev. B 100, 045117 (2019).

[110] A. W. Sandvik, J. Phys. A 25, 3667 (1992).

[111] A. W. Sandvik, Phys. Rev. B 59, R14157 (1999).

[112] O. F. Syljuåsen and A. W. Sandvik, Phys. Rev. E 66 , 046701 (2002).

[113] J. Skilling and R. K. Bryan, Mon. Not. R. Astron. Soc. 211, 111 (1984).

[114] M. Jarrell and J. Gubernatis, Phys. Rep. 269, 133 (1996).

[115] R. Steinigeweg, F. Heidrich-Meisner, J. Gemmer, K. Michielsen, and H. De Raedt, Phys. Rev. B 90, 094417 (2014).

[116] Since the tDMRG data in Ref. [81] are calculated for a finite temperature $T=10$ without taking into account the susceptibility $\chi$, we have rescaled the tDMRG data by a global factor for better comparison. Apparently the curvatures of tDMRG and DQT data agree convincingly for $\Delta=1$ (Fig. 1) and $\Delta=1.5$ (Fig. 2).

[117] J. Wurtz and A. Polkovnikov, arXiv:1808.08977.

[118] J. Richter, J. Herbrych, and R. Steinigeweg, Phys. Rev. B 98, 134302 (2018).

[119] D. L. Huber, Physica B 407, 4274 (2012).

[120] A. S. T. Pires and L. S. Lima, J. Phys.: Condens. Matter 21, 245502 (2009).

[121] W. Chen, K. Hida, and B. C. Sanctuary, Phys. Rev. B 67, 104401 (2003).

[122] A. Karahalios, A. Metavitsiadis, X. Zotos, A. Gorczyca, and P. Prelovšek, Phys. Rev. B 79, 024425 (2009).

[123] R. Steinigeweg, J. Herbrych, F. Pollmann, and W. Brenig, Phys. Rev. B 94, 180401(R) (2016).

[124] P. Prelovšek, M. Mierzejewski, O. Barišić, and J. Herbrych, Ann. Phys. 529, 1600362 (2017).

[125] S. Bera, G. De Tomasi, F. Weiner, and F. Evers, Phys. Rev. Lett. 118, 196801 (2017).

[126] F. Weiner, F. Evers, and S. Bera, Phys. Rev. B 100, 104204 (2019).

[127] M. Serbyn, Z. Papić, and D. A. Abanin, Phys. Rev. B 96, 104201 (2017). 